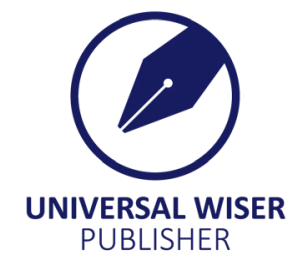

\title{
Survey on Recommender System Using Deep Learning Networks
}

\author{
Sushma Jaiswal ${ }^{1}$, Tarun Jaiswal ${ }^{2 *}$ \\ ${ }^{1}$ Guru Ghasidas Central University, Bilaspur (C.G.), India \\ ${ }^{2}$ Department of Computer Applications, NIT Raipur, Raipur, India \\ E-mail: tjaiswal_1207@yahoo.com
}

\begin{abstract}
In today's times, the recommended system is a very powerful weapon of shoppers that is very helpful in advancing the Internet, personalized tendencies, and online shopping. The recommended system is used primarily for commercial benefit. The recommended system works on the strength of the user's past shopping experience and its feedback, whether it is positive or negative. Hence the recommended system is also an innovative method. There is a deferred method of the recommended system which has its own advantages and disadvantages. In this paper, the recommender system based on deep learning is proposed, and also discussed the challenges and issues which are related to the deep learning based recommender system. i.e., Accuracy, Cold Start Problem, Scalability States etc. In this paper, we have also discussed the work done so far, which has been given by various scientists, researchers and investigators. Advancement of machine learning and deep learning is very big, in today's era. This study will help the Researcher to move forward.
\end{abstract}

Keywords: deep learning, recommender system, collaborative filtering, issues, personalized recommender system, modern recommender system

\section{Introduction}

Recommender systems (RS) have progressed hooked on an essential instrument for assisting user's variety cognizant pronouncements and adoptions, expressly Handling data in very large quantities is a very big problem, so handling data through a Recommender based method is a very good option. It has two options which are Content-based RS ${ }^{[1]}$ and collaborative filtering $\mathrm{RS}^{[2]}$. Scientists gave the validation and effectiveness of both methods.

In paper ${ }^{[3]}$ through the prodigious attainment of deep neural networks (DNNs) in numerous pitches, in recent times, investigators have suggested numerous DNN centered factorization prototypical to find out mutually minor- and elevatedorder feature exchanges. Notwithstanding the authoritative capability of learning a capricious function commencing information, basic DNNs produce feature communications discreetly and at the bit-wise glassy. In this investigations, authors recommended an innovative Compressed Interaction Network (CIN) which provide feature communications in an unambiguous style and at the vector-wise glassy. Authors also conglomerate a CIN and a traditional DNN into one amalgamated prototypical, and so-called this novel prototypical eXtreme Deep Factorization Machine (xDeepFM). And the $\mathrm{xDeepFM}$ is intelligent to learn convinced bounded-degree feature communications unambiguously; it can be able to learn indiscriminately minor- and elevated-order feature communications discreetly.

RS are a spontaneous stroke of protection alongside shopper over excellent. Prearranged the volatile development of data accessible on the net, customers are habitually received with supplementary than uncountable merchandises, cinemas or cafeterias. As such, personalization is an indispensable approach for smoothing improved user involvement. Altogether, these arrangements have been frolicking an energetic and crucial protagonist in numerous data entrance schemes to enhancement trade and expedite policymaking procedure ${ }^{[4,5]}$ and are unescapable crosswise abundant net areas named as e-commerce and media websites.

On the new influences, deep learning (DL) representations in recent times demonstrated that excessive perspective for learning active demonstrations and distribute state-of-the-art enactment in the field of computer vision ${ }^{[6]}$ and natural language processing ${ }^{[7]}$ domains. In DL prototypes, features are learned in a supervised or unsupervised fashion. While they are additionally likeable than shallow representations in that the features can be learned robotically (e.g., operative feature demonstration is learned from text contented), they are low-grade to shallow representations such as CF in 
apprehending and learning the resemblance and inherent association amongst items. This sounds for mixing DL with CF by accomplishment DL collaboratively.

\section{Related work}

The authors presented a constrained variety of the Probabilistic Matrix Factorization (PMF) prototypical that is grounded on the supposition that users who have evaluated comparable groups of movies are probable to have similar predilections. The consequential prototypical is intelligent to generalize significantly healthier for users with exact few assessments. While the forecasts of manifold PMF representations are linearly joint with the forecasts of Restricted Boltzmann Machines representations, the authors attain an error rate of 0.8861 , which is closely $0.7 \%$ healthier than the notch of Netflix's specific scheme.

The most important methods for RS broad-spectrum scheme is Gaussian Process Factorization Machines (GPFM) suggested in [9]. By presenting Gaussian processes, complex, non-linear user-item-context collaborations are able to apprehended and therefore foremost to flexible demonstrating capability. The learning is ended through SGD that gauges linearly by the entire amount of explanations and therefore, construction GPFM ascendable to huge datasets. It is appropriate to together the explicit feedback surroundings and the implicit feedback surroundings, in which circumstance it is entitled GPPW (GPFM-based pairwise preference model). GPPW is imitative by varying its covariance function and as the variation of GPFM for pairwise item positioning through implicit feedback. The exact topical and the greatest momentous investigation prepared by Balazs Hidasi (supervised by Domonkos Tikk) ${ }^{[10]}$. The technologically advanced tensor factorization approaches iTALS ${ }^{[18]}$ and iTALSx ${ }^{[11]}$, as fine as the GFF (General Factorization Framework) ${ }^{[12]}$. Together iTALS and iTALSx are tensor factorization approaches that usages pointwise positioning through enhancing for weighted summation of squared mistakes. iTALS evaluations partialities are exhausting the N-way communication prototypical. While iTALSx assessments predilections exhausting the pairwise communication prototypical. So, approaches for implicit feedback that be able to arrange by the illogical and huge quantity of context measurements are GPFMbased pairwise predilection prototypical (GPPW) ${ }^{[9]}$, iTALS ${ }^{[13]}$ and General Factorization Framework (GFF) ${ }^{[14]}$. It would be motivating to scrutinize them all, nevertheless, merely GPPW's enactment is visibly obtainable, and the enactment of iTALS and GFF are padlocked contained by the specific company. Consequently, the foremost contender technique is GPPW. The approaches for explicit feedback that be able to treaty by the uninformed and huge amount of context measurements are Multiverse Tensor Factorization (Multiverse TF) ${ }^{[14]}$, Context-aware matrix factorization (CAMF) ${ }^{[15]}$, Factorization Machine (FM) ${ }^{[16]}$ and GPFM ${ }^{[9]}$. While approaches for explicit feedback grounded CARS frequently can't be unswervingly castoff for implicit feedback, by arbitrarily specimen negative items for positive items agreed user-context conformation, FM and GPFM be able to be castoff for an implicit situation as the research of GPFM ${ }^{[9]}$ recommends. Though, subsequently, GPPW is at present the pairwise predilection different of GPFM and the research moreover demonstrated that GPPW is further appropriate for implicit feedback. Pairwise Interaction Tensor Factorization (PITF) ${ }^{[17]}$ is formerly premeditated on behalf of personalized label RS. It crumbles a 3-D assessment tensor hooked on 03 feature matrices.

Bayesian Personalized Ranking (BPR) ${ }^{[18]}$ is a pairwise positioning tactic. It samples the negative response for each positive response, i.e., for each occasion of the customer, and it obtain the samples from an item which has not any deal to the user. And this is expected that user favors those item more frequently which he used in past. The optimization standard is maximum posterior expectations that are consequentially commencing a Bayesian analysis of the delinquent.

\section{Background}

Although a RS is itself a well-established system that is used exclusively for data filtering. Deep-learning is a subfield of the machine learning. And both of these ventures are such that they have the ability to solve any traditional or modernized problem easily and that is the reason why this subject has also attracted the scientists of computer science. In this section, we have discussed the recommended system and its numerous types and issues and challenges.

\subsection{Recommender system}

In the recommended system, the AIM of the shopper is how to keep the item and product in mind, increasing the benefit, along with user feedback. Suggestions for movies, Netflix, amazon's books, music/video songs are reallife examples of the recommended system which increases the strength of the industry. It all depends on some special information received from the user, which is on old Experiences of purchasing. In this, the order of 1 to 5 is given to know the feedback of the user, 1 means the quality of the product is not correct, and the order of 5 indicates that it is the 
best product. This review is user and product-specific. The recommended system supervises this information and plays an essential part in advancing the user and item to this supervised idea.

Collaborative Filtering (CF) schemes examine past communications alone, while Content-based Filtering (CBF) schemes are grounded on profile traits; and hybrid Filtering techniques (HF) effort to cartel together of these strategies. The construction of RS and their assessment on actual difficulties is a vigorous extent of the investigation.

\subsection{Personalized recommender system}

In this kind of RS, the scheme objectives at recommending users anticipated items constructed on their past performance also the personal connection of social nets by allowing for 03 perceptions, namely as Interactive influence, Attention circle descent, User separate attention.

The resulting stage is to identify a method contingent on the domain. Diverse methods are revealed in Figuer 1. There are various arrangements of RS methods, and the greatest accepted one is well-defined by the Burke as followed ${ }^{[19]}$ :

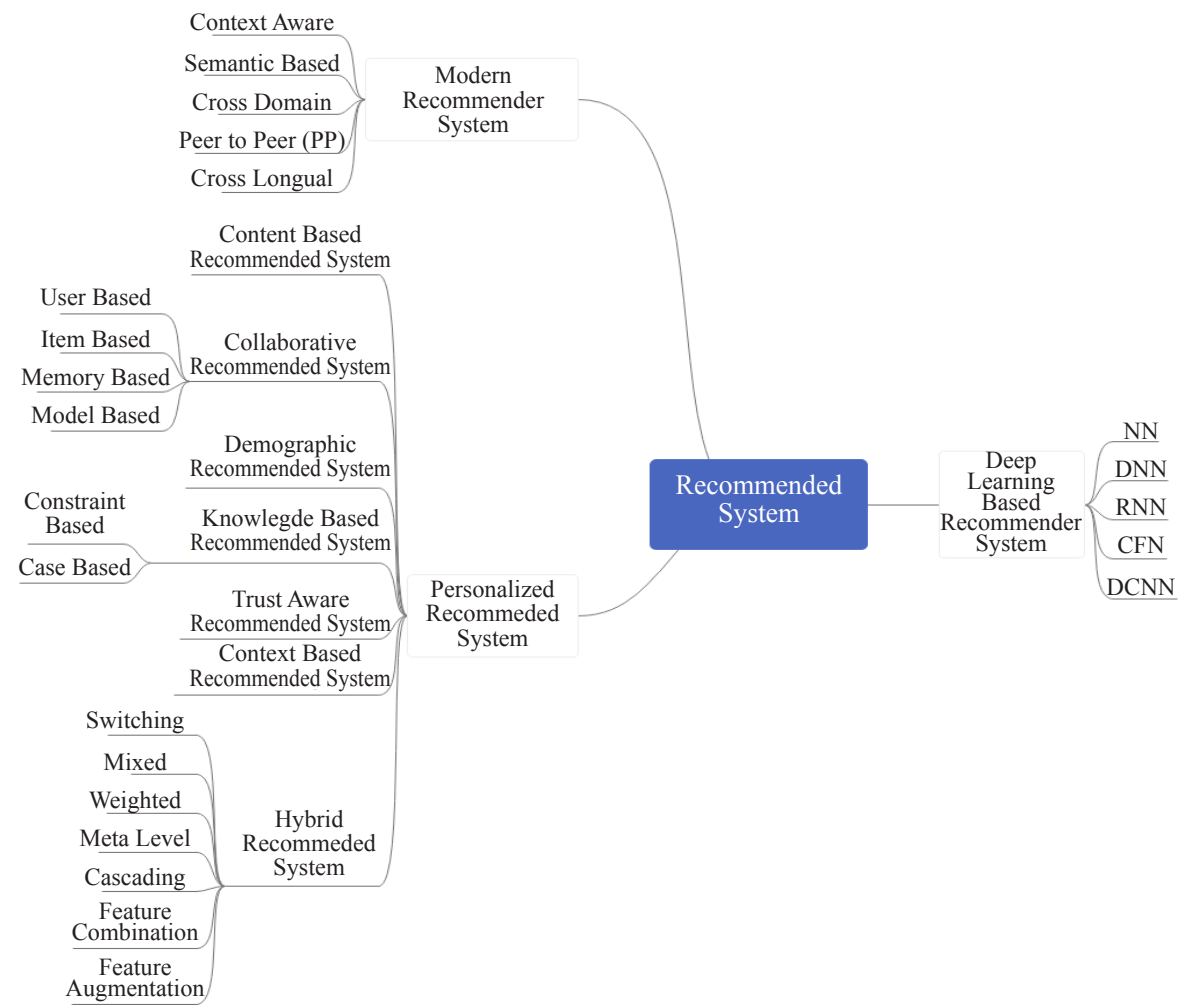

Figure 1. Recommender system organization in design \& development stages

Content-based system: In this system, the RS examines a given set of item's explanation that has been graded earlier by the user. Thereafter, it dimensions a user prototypical or user interest profile conferring to the features of the valued objects and competitions and the characteristics of the profile alongside qualities of a content of a thing. Therefore the scheme adopts to recommend the things to persons who adored them previously also. For instance, if a funny movie obtains the positive score by the user, then the scheme adopts to recommend further movies fitted in a similar group.

Filtering using the collaborative approach: In this investigation, the author's attentiveness on CF-based communal RSs, since furthermost prevailing communal RS are CF-based. Resulting the cataloguing of old-fashioned CF-based RSs ${ }^{[20,21]}$, the authors pigeonhole CF-based communal RSs into 02 broad types: Matrix Factorization (MF) cantered communal recommendation methodologies, and Neighborhood cantered social recommendation approaches. In MF-based social recommendation methodologies, user-user communal belief data is combined with user-item response antiquity (e.g., ratings, clicks, and procurements) as to progress the accurateness of old-fashioned MF-based RSs, which first factorize user-item response information. Neighborhood constructed communal recommendation tactics comprise Social Network Traversal (SNT) constructed tactics and Nearest Neighbor tactics. An SNT-based procedure produces a recommendation for a user afterwards crisscrossing and enquiring their undeviating and unintended networks in their neighborhood in the communal network. ANN method combines the old-fashioned CF neighborhood by communal neighborhood, and forecasts assessments of items or recommends a list of items ${ }^{[2]}$. CF is the greatest extensively recognized and used in RS. This 
method recommends things to the consumer that were enjoyed in the past by different consumers with similar perceptions. The recommendations are fashioned centered on ratings or usage decorations deprived of any essential of energetic data about users or items. The subsequent 04 filtering tactics are deliberated in CF:

User-based collaborative filtering deals with the correlation concerning sets of users. This technique has been implemented in manufacture superior forecasts and recommendations, however, it is unsuccessful in exercise for online uses. It is too sluggish while handling the millions or a huge number of users ${ }^{[23]}$.

Item-based collaborative filtering is a substitute technique, conveying correlations concerning items and recommending items with great resemblance to the set of items previously ranked by the customer. The item-based procedures are wont to be quicker in terms of online reply time than the user-based procedures, precisely, if the item associations are pre-computed. The item-based procedure, which also outbursts agreeably into unary score sets, rapidly developed widespread in moneymaking presentations ${ }^{[23]}$. The datasets which contain very few data / no data are known as Unary datasets.

Memory-based systems effort single with the matrix of user-item assessments and use any rating engendered previously the recommendation procedure. They typically use resemblance metrics to the extent the distance concerning 02 users or 02 items, grounded on separately of their ratios. Memory-based approaches suffer from scalability difficulties, since it is essential for progression to create the single forecast from the entire information that needs heavy resources of computing and create the progression period overwhelming ${ }^{[24]}$.

System built on model: This model produces the prototypical expending gained data for recommendation generation. Model-based on these schemes are time-consuming in its initial stage / preprocessing stage, but when the prototypical is generated, recommendations are generated instantly. The system built on a model may have some drawbacks as well. Approximation multidimensional limits are provided by the few diverse models. This primes to great warmth in terms of data variations. There might be a chance of prototypical mismatching with data, which is accountable for an inappropriate recommendation, since not any hypothetical prototypical has the impending to be pragmatic in actual applications ${ }^{[25]}$.

Demographic: On the basis of user's personal characteristics, the demographic recommender structure provide the recommendation on the profile of demo-graph. The demographic recommender structures categorize the users dependent on their individual characteristics to deliver recommendations on demographic profiles. The fundamental philosophy is that diverse demographic positions must obtain their specific compromises. This kind of recommender structures perform someway analogous to content-based, but the advantage over this method is that unlike collaborative and content-based methods, it does not unavoidably essential user ratings antiquity ${ }^{[26]}$.

Knowledge-based: this method emphases on knowledge bases that are not exposed by content-based and collaborative filtering methods. It produces recommendations based on precise domain information of the users 'necessities, the items' features and how these features can encounter users' wants and penchants. This method is apt to act healthier than the other methods in the commencement of their service as they do not use any scores. Though, in instruction to retain this preeminence, they should be prepared with learning mechanisms to achievement the human / computer communication records ${ }^{[27]}$. There are 02 arrangements for the knowledge-based recommendations:

Case-based: By using the resemblance matrices, it recovers the items, which is similar to the user's desire.

Constraint-based: By using some common recommendation rules, it identifies the objects.

Context-based: It emphases on appropriate data with time, place, wireless instruments and so on. The appropriate data may be composed of either explicit or implicit feedback.

Trust-aware (community-based): This scheme reflects the user's preference network for its recommendation. It is usually confessed that persons be apt to receive the recommendations from their networks somewhat than from analogous unidentified persons ${ }^{[29]}$. The everyday emergent admiration of social nets elevated the attention in Communal-based RS, also named as social RS. In wide-ranging, the arrangement gets data around the social dealings of the user and her friends' predilections and assessments to deliver its recommendations.

Hybrid: This RS conglomerate the upstairs declared methods to attain greater recital. A hybrid recommender system, merger 02 methods, and efforts are used to fix the shortcoming of others. For instance, CF is not intelligent to grip new items deprived of any ratings, though the content-based method do not have any kind of difficulties with fresh objects meanwhile the recommendations are grounded on the items features which are simply obtainable. Various approaches for hybridization are prevalent:

Weighted hybrid: This method uses a linear formulation to association scores of apiece recommendation constituent. Therefore, mechanisms must have the capability to engender recommendation notches which are linearly combinable.

Switching hybrid: In this method, the structure chooses a single recommendation method amongst other applicants. 
There may be dissimilar assortment standards, like assurance assessment or exterior standards, dependent on the knowledgeable condition. All of them constituent might have a dissimilar concert in various circumstances.

Mixed hybrid method: This method depends at amalgamation and awarding compound ranked gradients. Therefore, mechanisms must use the essential procedure to generate recommendation slants with ranks that can be amalgamated into a single ranked list. The key problem is exactly how to produce the new rank notches.

Feature combination hybrid method: This technique has the two dissimilar recommendation gears that are contributing $\&$ actual recommender. The first one constitutes enclosures structures of one source, and the other constitutes a source of the real recommender. Real recommender work on the available data which is amended by another.

Feature augmentation hybrid method: This method is analogous to feature amalgamation hybrid, although this technique is more bendable as well as it also adds reduced proportions since the provider yields novel features.

Meta-level hybrid method: This method consist of two-component and its main component use prototypical which is generated via next as input and this is quite dissimilar from the feature intensification, that use the learned prototypical for generating features as an input for the subsequent algorithm. In the above said method, however, whole prototypical provide input to the other one.

Cascade hybrid method: This method consumes the single recommendation element for harvesting the ranked list thereafter, it consumes another element to improve the recommendation list.

\subsection{Modern techniques}

Context-Aware: It usages contextual data like withstand estimating, day-night time etc. Utmost of RS appliance this method for professional deployment of the information e-commerce industry, social nets etc.

Semantic Based: This sympathetic method inhabit the internet in the form of metaphysics. Numerous RS show their performance regarding semantic approaches like trust management, policymaking, social communication groups etc. ${ }^{[30]}$.

Cross-Domain Based: Also recognized as linked-domain recommendations. This practice comprises 03 necessary plugs a) domain knowledge transfer b) user-item overlap c) recommendation generation. It exploits the knowledge learned from the source to provide recommendations to target ${ }^{[31]}$.

Peer to Peer (PP): This method has the ability to resolve the more substantial scale matter, at this time, each peer cluster which has the same perception associated to another dedicated, concerned peer.) It exploits the elementary antiquity to provide recommendations ${ }^{[32]}$.

Cross-Lingual: It usages vocabularies, machine translation for data retrieval. Various classification approaches are castoff for this method ${ }^{[33]}$. The chore of this tactic to assist the user in discovery the text, document, news etc. in the language he or she identifies rather than the source language ${ }^{[34]}$.

\section{Issues and challenges}

The recommender system is an effective system that provides results to various clients according to their advantage. Step by step instructions to recommend the new user, is a main fundamental issue of the recommender system. Again there is a significant amount of work that has been done to solve this problem and recommend a new user based on their interest. 


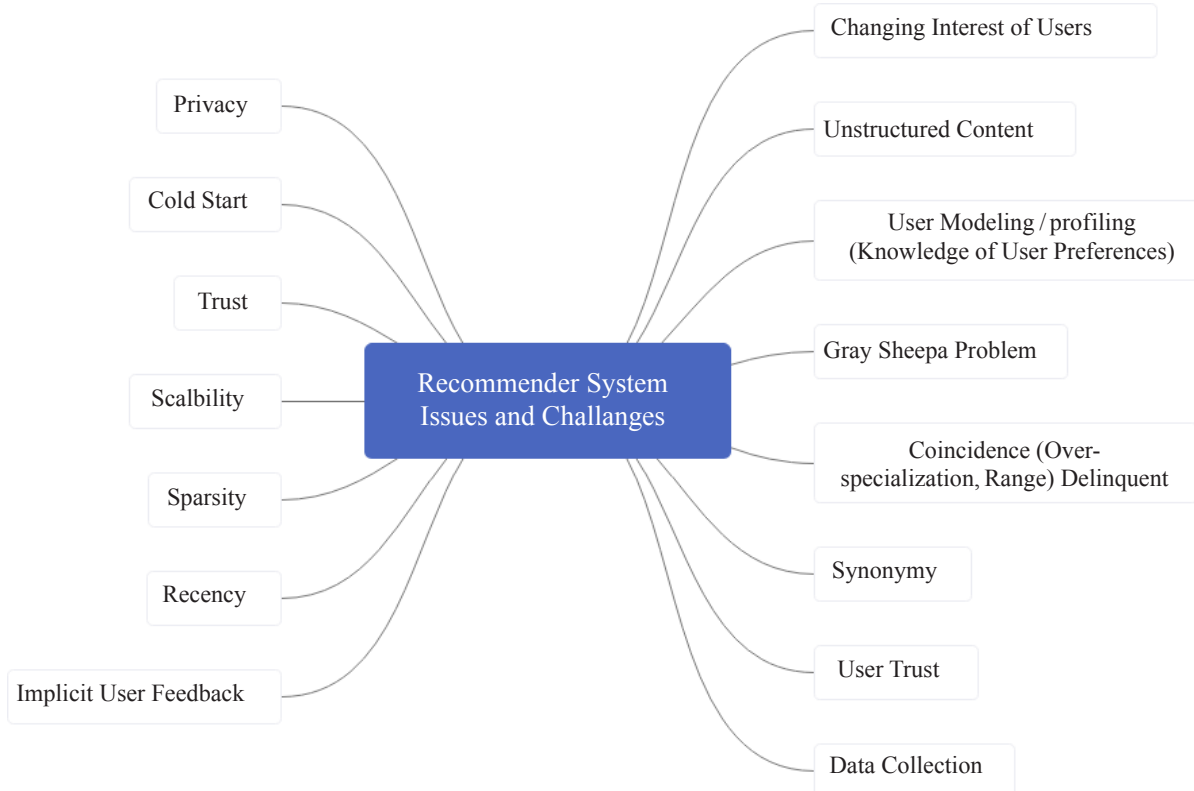

Figure 2. Recommender system issues and challenges

Cold-start: It's burdensome to stock-supply tenders to novel consumers as his profile is considerable unoccupied and consumer has not reviewed every object nevertheless consequently consumer trend is incomprehensible to the outline. This can be so-called the cold begin problem. In roughly recommender backgrounds this problem is esteemed with summary although fashioning a profile. Things can similarly have a frigid begin once they element of dimension novel at interludes the background and ought to been estimated a while recently. Every of complications square extent typically in accumulation comprehended with hybrid methods.

Trust: The thoughts distinct persons by quick antiquity won't be that suitable as a consequence of the opinions of the persons WHO have fashioned antiquity in their summaries. The delinquent of faith materializes concerning valuations of a designated consumer. The exertion is also elucidated by diffusion of must the consumers ${ }^{[35]}$.

Scalability: With the development of quantities of clients and things, the framework wants additional assets for preparing data and shaping proposals. Larger part of assets is overwhelmed by the motivation behind deciding clients with comparative tastes, and merchandise with comparable portrayals. This issue is also tackled via blend of different sorts of channels and physical change of frameworks. Parts of different calculations may likewise be executed disconnected with a specific end goal to quicken issuance of suggestions on the web.

Sparsity: In on-line retailers that have a huge live of procurers and things their square measure very often purchasers that have appraised a handful of things solely. Employing community and different procedures recommender frameworks by and large make the neighbourhood of clients utilizing their profiles. On the off chance that a consumer has evaluated a couple of things solely then, it's truly exhausting to make your mind up his style, and he/she may be recognized with the inappropriate neighbourhood. Insufficiency is that issue of absence of data ${ }^{[36]}$.

Privacy: Defense has been the primary crucial subject. Observance in mind the end objective to get the most accurate and right recommendation, the outline must improvement the most extent of data plausible about the client, with statistic data, and knowledgeable data about the realm of a specific consumer. Really, the subject of steadfastness, security \& privacy of the information arises. Numerous on-line passages suggested convincing insurance of fortification of the procurers by discrimination exact calculations and developments.

Recency: Recency is one the most significant defies in news recommendation area. Most of the user's neediness to read fresh news in its place of old dated works. So the prominence of news items reductions in time. On the other hand, some news articles may be associated with each other that the user may neediness to read the preceding news items connected to the one he / she already reads or he/she may neediness to retain conversant around that subject ${ }^{[37]}$.

Implicit User Feedback: User comments are moderately significant to make more detailed recommendations. Deprived of explicit responses, it may not be conceivable to understand if the user enjoyed the investigation, whereas persons read or not ${ }^{[38]}$. Nonetheless, it is not concrete for the arrangement to interrelate with the user uninterruptedly. So the system should be intelligent to collect implicit feedbacks effectively while defensive the user confidentiality. 
Changing Interests of Users: Alternative important encounter is envisaging the imminent safeties of users for healthier recommendations as persons may have fluctuating safeties ${ }^{[39]}$. For some areas like movie or book recommendations, the variation of user interest occurs more gradually. Nonetheless, for the news area, it is actually solid to forecast the vicissitudes. Likewise, some persons may read the news, not as he/ she attentive in the theme is universal, but as she found it significant.

Unstructured Content: For the structures which require contented data, it is stiff to investigate the content, particularly for the news area. For healthier news recommendations, news items should be controlled and machine legible ${ }^{[40]}$.

User Modeling / profiling (Knowledge of User Preferences): User profiling is an imperative constituent of RS. To make further specific recommendations, it is required to build a user profile. As it is specified in [40,41], there are various methods for user profiling.

Gray Sheep Problem: Since CF recommends items conferring to the user's common benefits with other users, it is not conceivable to recommend appropriate items to people whose favorites do not unswervingly decide or distress with any assemblage of people ${ }^{[42]}$. When the entire number of users upsurges, the prospect of this problem happening diminutions ${ }^{[43]}$.

Coincidence (Over-specialization, Range) Delinquent: This is the delinquent when the method recommends analogous or similar items with the already recommended ones. For the newscast area, a news item written inversely in different news sources may be recommended by the RS as different researches. This is evident that the user won't be satisfied if he obtains the identical recommendation. The system should always be intelligent to determine new items to recommend by circumventing the same items. In [44] the delinquent is discoursed in facet for content-based arrangements, but it is also a delinquent for CF systems ${ }^{[43]}$.

Synonymy: Same items can be named contrarily by detached resources, and it is not possible for mechanisms to understand that they refer to the same item. For example; even if the "child's movie" and "child's film" have the same sense, they can be preserved as different items by the RS ${ }^{[42]}$.

User Trust: The user who has less history may be weakly associated with the shopping summary while the other who have a strong history is associated firmly with the shopping summary. This issues can be solved by the precedence distribution ${ }^{[45]}$.

Data Collection: In two ways, it can be completed either implicitly or explicitly. The data which is explicit in nature is delivered by the user, i.e. user effort. While the data which is implicit in nature is composed of effortlessly reachable sources, like user profile data, which is not consciously delivered via user ${ }^{[46]}$.

\section{Deep learning based recommendation systems}

In this paper, author ${ }^{[47]}$ presented a latent factor structure for recommendations and solve the cold start problem for music audio when they cannot be attained from convention facts. Authors equated the old-fashioned tactic expending a bag-of-words demonstration of the acoustic signals by deep convolutional neural networks (DCNN), by using masses of song dataset estimate the forecast quantitatively and qualitatively. Latent factor harvest the serviceable recommendations notwithstanding details that there is a big hole concern the physiognomies of a song the distress the user predilection and the conforming acoustic signal. DCNN expressively overtaking the old-fashioned tactic.

Collaborative filtering (CF) ${ }^{[48]}$ has been extensively engaged inside recommender systems (RS) to resolve numerous practical glitches. Wisdom of actual Latent factors king in CF. The old-fashioned CF approaches used matrix factorization techniques knowledge the hidden factors from the user-ratings and its conceive the cold start difficulties and the sparsity delinquent.so the authors proposed universal deep construction for CF by assimilating matrix factorization through deep feature learning. They deliver a usual instantiations framework by coalescing probabilistic matrix factorization thru marginalized denoising stacked auto-encoders. The collective background central to an ungenerous fit over the latent features as designated by its enhanced presentation in judgement to preceding advancement representations terminated 04 great datasets for the chores of movie/ book commendation and reaction forecast.

Authors ${ }^{[49]}$ tried to explain the cold start difficulty in CF, Enthused by the allied effort, authors, train a NN on semantic cataloguing material as a comfortable prototypical and using it as a preceding in a CF prototypical. Such an arrangement immobile permits the user eavesdropping information to "speak for the situation". The projected scheme is assessed on the Truckload Song Dataset and demonstrations comparably healthier consequence than the CF approaches, in accumulation to the promising recital in the cold-start incident ${ }^{[49]}$.

Authors ${ }^{[50]}$ investigated the NN have not been extensively deliberate in CF. So limited paper was available on NN with Netflix Prize. Whereas DL incredible triumph in image and language recognition, sparse inputs acknowledged a smaller amount of courtesy and leftovers an inspiring delinquent for NN. However, the Sparse responses are precarious for 
CF. In this paper, authors present a NN construction which calculates a non-linear matrix factorization from sparse rating inputs and demonstration practically proceeding the movieLens and jester dataset that show that the investigated technique accomplishes as well as the finest CF procedures. For this purpose, the authors used a reusable plugin for Torch and a prevalent NN structure.

Establish ${ }^{[51]}$ that the enlarged elasticity obtainable by the product-of-experts prototypical permitted it to accomplish advancement routine on the Amazon assessment dataset, overtaking the latent Dirichlet allocation (LDA)-based tactic. Nevertheless, stimulatingly, the superior demonstrating influence obtainable by the RNN seems to weaken the representation's capability to deed as a regularizer of the artefact demonstrations.

Authors investigate ${ }^{[52]}$ a content Based Recommendation scheme to discussed quality as well as scalability. Authors investigate to use an amusing feature customary to characterize users, conferring to their glancing antiquity and examine inquiries. They give the expenditure a Deep learning method collects the feedback given by the user, where the potential-feedback between moderator and their favored gears is browbeaten. Author's investigated by slant on 03 actual recommendation schemes learnt from dissimilar foundations. The summed-up of the suggested methods work better than the other.

In [53] today 'high end technology allow us to capture the series of consumer action at the exclusive scale. Even though such commotion fuels are lavishly obtainable. In divergence to obvious assessments, such activity logs can be composed in a non-intrusive technique and can compromise wealthier acumens into the undercurrents of consumer inclinations, which might hypothetically principal of further precise user representations. Authors investigated a lavishly obtainable data and, by coalescing thoughts starting latent factor representations for CF and language demonstrating, recommend an innovative, stretchy and communicative collaborative categorization prototypical built on RNN. The proposed prototypical is intended to detention a user's contextual stage as an adapted hidden vector by succinct signs as of a data-driven, consequently variable, previous period stages No.'s, and characterizes substances by a real-valued implanting. Authors set up that, by manipulating the inherent configuration in the information, proposed construction chiefs to a competent and real-world technique. Additionally, authors determine the adaptability of exemplary by smearing it to 02 altered chores: music recommendation and mobility forecast, and summed-up the empirically that proposed prototypical unfailingly beat static and non-collaborative approaches.

In this investigation ${ }^{[54]}$, the proposed technique profitable to the usage of deep recurrent neural networks to encrypt the text categorization into a latent vector, exactly gated recurrent units (GRUs) accomplished endwise on the CF chore. For the chore of methodical tabloid endorsement, this harvests representations with suggestively advanced accurateness. In cold-start circumstances, the proposed method tired out the preceding advancement, all of which overlook expression directive. The recital is additionally enhanced by multi-task learning, where the text encoder net is trained for a mixture of content recommendation and item metadata forecast. This normalizes the CF prototypical, enriching the problematic of the sparsity of the pragmatic assessment matrix.

In this paper, the author investigated ${ }^{[55]}$, Collaborative Filtering Neural network architecture aka CFN, which calculates a non-linear Matrix Factorization as of sparse rating inputs and side evidence. Author's demonstration experimentally on the MovieLens and Douban dataset that CFN beats the techniques at that time and profits from side data. Authors deliver an enactment of the procedure as a returnable plugin for Torch, a prevalent NN framework.

In this paper, the author proposed ${ }^{[56]}$ a NN construction, aka CFN, to implement CF with contiguous data. Conflicting to extra endeavors with $\mathrm{NN}$, this joint Network participates side data and learn a non-linear demonstration of users or items into an inimitable NN. This tactic accomplishes to together rhythms of techniques at that time, results in CF and comfort the cold start problematic on the MovieLens and Douban datasets. CFN is also accessible and healthy to deal with great size dataset. Authors ended numerous privileges that Autoencoders are meticulously accompanying to low-rank Matrix Factorization in CF. To end with, refillable source code is delivered in Torch and hyper-parameters are in case to replicate the consequences.

Authors announced Meta-Prod2Vec ${ }^{[57]}$, a new item implanting technique that improves the prevailing Prod2Vec technique by item metadata at the training period. This effort created an innovative linking concerning the current embedding-based approaches and consecrated Matrix Factorization approaches by familiarizing learning with lateral data in the background of embeddings. The authors summed-up that the Meta-Prod2Vec constantly outperforms Prod2Vec together comprehensively and in the cold-start establishment, and that, once pooled through a standard CF tactic, overtakes all other confirmed approaches. These consequences, composed with the condensed enactment budget and the circumstance that proposed technique does not touch the operational recommendation construction, varieties this explanation gorgeous in circumstances where item embeddings are now in use. 
Memorization of feature partnerships ${ }^{[58]}$ in excess of a widespread accustomed of cross-product feature alterations are operative and interpretable and simplification cast-off for large-scale regression and classification glitches with sparse inputs, reciprocally noteworthy for RS. Authors proposed an extensive and DL schema together with the powers of Memorization \& generalization types of architecture. Authors productized and assessed the agenda on the RS of GooglePlay, a vast level of profitable app accumulation. Operational experimentation consequences exhibited that the Extensive \& Deep model headed to noteworthy enhancement on app attainments in excess of wide-only and deep-only representations. Authors used open-sourced proposed execution in TensorFlow.

Authors suggested ${ }^{[59]}$ a hybrid RS that deliberates actual users data and elevated depiction for audio information. For this purpose author used a DL procedure, CDNN describes for an audio piece in a space. And authors examine the methods with comparisons of various past researcher's methods. The envisioned hybrid music recommender beats the predictions associated with an old-fashioned content-based recommender.

In this study, the author applied a ${ }^{[60]}$ generous-of-contemporary-recurrent-neural-network (GRU) to innovative presentation area: RS. Authors selected the assignment of term centered recommendations, since it is an almost significant capacity, but not well investigated. Authors improved the rudimentary GRU in directive to appropriate the chore healthier by familiarizing session-parallel mini-batches, mini-batch founded productivity specimen and ranking loss function. Authors exhibited that projected technique can suggestively overtake prevalent standards that are cast-off for this chore.

In this paper, authors ${ }^{[61]}$ propose CF-NADE, a feed-forward, autoregressive architecture for collaborative filtering tasks. CF-NADE is motivated by the influential work of RBM-CF and the modern encroachments of NADE. In this paper, the authors proposed to serve restrictions between diverse assessments to expand the enactment. Authors also designate a factored account of CF-NADE, which decreases the amount of restrictions by factorizing a large matrix by a product of 02 lower-rank matrices, for improved its mapping. Furthermore, authors yield the ordinal environment of predilection into deliberation and suggest an ordinal budget to heighten CF-NADE. Lastly, the prolong CF-NADE to an unfathomable prototypical by the adequate upsurge of computational involvedness. For this purpose author used 03 standard datasets and summed-up CF-NADE beats the state-of-the-art approaches on CF chores.

In this paper ${ }^{[62]}$, described DNN construction for recommending YouTube videos, divided into 02 different glitches: candidate generation and ranking. The proposed deep collaborative filtering prototypical is intelligent to meritoriously integrate numerous signals and prototypical their collaboration with coatings of complexity, beating preceding matrix factorization tactics. The proposed tactic accomplished much improved on watch-time prejudiced ranking assessment metrics likened to foreseeing click-through rate unswervingly.

Authors examined ${ }^{[63]}$ how these features can be browbeaten in RNN grounded gathering representations using DL. Authors demonstrated that noticeable methods do not influence these data cradles. Hence authors introduced the sufficient number of parallel RNN (p-RNN) constructions to model sessions grounded on the clunks and the features (images and text) of the clacked items. The proposed substitute training policies for p-RNNs that ensemble they improved than customary exercise. The proposed p-RNN constructions with appropriate training have substantial routine perfections terminated feature-less assembly representations while all session-based representations beat the item-to-item type standard.

RNNs remained ${ }^{[64]}$ in recent times projected for the session-based recommendation chore. The representations exhibited auspicious enhancements over old-fashioned recommendation methods. In this revision authors, demonstrated RNN-based for session-based recommendations, and investigated the presentation of 02 procedures to expand model enactment, viz., data augmentation, the methods give an entered data swingness. It is also a comparative and innovative work for furcating.

An investigation based on the RecSys Challenge 2015 dataset validate comparative enhancements of $12.8 \%$ and $14.8 \%$ in excess of formerly conveyed consequences on the Recall@20 and Mean Reciprocal Rank@20 metrics correspondingly.

By using fusion methods ${ }^{[65]}$ categorically advancement in the presentation. Expending transformation and dot product of the song demonstrate vectors (final LSTM hidden state) assistance progress the concert by a proportion, together the lyrics prototypical and the audio based prototypical. This demonstrates that there is data accumulated in those man oeuvres. For the lyrics prototypical, additional FC layer does not improve much and correspondingly for the audio prototypical, it is vibrant that consuming one convolution layers is healthier than consuming 02 convolutional layers. This demonstration together the influence of the mixture procedures to imprisonment greatest of the data and that music can be recognized by observing at the instant neighbourhood in the time-domain. In this research, the consequences demonstrate sufficient resistant that expending Lyrics and Audio unaided is adequate to categorize songs as alike or disparate. 
In this paper ${ }^{[66]}$, the authors presented the Collaborative Denoising Auto-Encoder (CDAE) for the top-N recommendation delinquent. CDAE acquires scattered demonstrations of the users and items via expressing the useritem reaction data by means of a Denoising Auto-Encoder construction. Numerous prior exertion can be perceived as superior suitcases of the projected prototypical. Authors directed a wide-ranging customary of tests on over three data sets to identify how the excellent of the prototypical apparatuses influence the enactment. The authors equated CDAE beside numerous further state-of-the-art top-N recommendation approaches and the consequences demonstration that CDAE beats the break of the approaches via a great brim.

The authors suggested ${ }^{[67]}$ a prototypical, so-called Deep Cooperative Neural Networks (DeepCoNN), contains 02 parallel NN joined in the most recent coatings. In the proposed method, different learning networks works, one of the attention on user actions misusing assessment by the user, and further network trained for items properties commencing the reviewers. So shared layer is presented for these 02 organized networks. The practically exhibit that DeepCoNN suggestively beats all starting point RS on a diversity of datasets. The authors summed-up, in evaluation with state-ofthe-art standards, DeepCoNN accomplished 8.5\% and 7.6\% enhancements on datasets of Yelp and Beer, correspondingly. On Amazon, it beat all the starting point and grown $8.7 \%$ enhancement on middling. Inclusive, $8.3 \%$ enhancement is accomplished by the suggested prototypical on all 03 datasets. DeepCoNN achieves a further reduction in MSE than MF. Particularly, once a solitary one assessment is obtainable, DeepCoNN achievements the extreme MSE reduction.

In this investigation ${ }^{[68]}$, authors recommend an innovative ranking tactic for $\mathrm{CF}$ based on $\mathrm{NN}$ that together studies a novel depiction of consumers and things in an entrenched planetary as well as the predilection next of kin of consumers ended couples of things. The projected prototypical is by environment appropriate for mutually imbedded and unequivocal response and contains the assessment of solitary precise limited constraints. From side to side widespread tryouts on numerous actual benchmarks, together unequivocal and imbedded, the authors demonstration the attention of learning the predilection and the implanting instantaneously when associated with learning those disjointedly. They moreover determine that proposed tactic is precise modest by the best state-of-the-art CF performances projected autonomously for an unequivocal and imbedded response.

The proposed prototypical ${ }^{[69]}$, termed as TransNets, spreads the DeepCoNN prototypical by familiarizing a surplus hidden layer expressive the board user-target item pair. So then regularize this layer, at exercise period, to be related to the additional latent depiction of the board user's assessment of the target item. Experimentally the proposed methods, show that TransNets and extensions of it advance significantly terminated the preceding state-of-the-art approaches.

In this investigation ${ }^{[70]}$, authors suggested to consume the clue of Denoising Auto-Encoders (DAE) to confrontation for cracking the data sparsity problem and degrade recommendation performance delinquent. Specifically, the authors suggested an innovative DL prototypical, the Trust-aware Collaborative Denoising AutoEncoder (TDAE), to learn condensed and operative depictions as of mutually grading and faith information for an uppermost-N recommendation. In specific, the contemporaneous an innovative NN through a weighted hidden layer to stability the prominence of these demonstrations. Additionally, the authors suggested an innovative correlative regularization to channel dealings amongst user predilections in changed viewpoints. Authors likewise comportment wide-ranging investigates on 02 communal datasets to associate with numerous state-of-the-art tactics. The consequences validate that the projected technique suggestively beats other evaluations for uppermost-N recommendation chore.

To address the cold-start challenges ${ }^{[71]}$, the authors suggested the probabilistic-modeling tactic entitled NeuralSemantic-Personalized-Ranking (NSPR) to cleave the supremacies of DNN and pairwise-learning. Precisely, NSPR compactly pairs a latent factor model by a DNN to learn a vigorous feature demonstration as of together implicit feedback and item contented, subsequently countenancing the proposed prototypical to generalize to unnoticed items. The authors validate NSPR's adaptability to assimilate numerous couple-segment probability functions and suggest 02 alternatives grounded on the Logistic and Probit functions. The author's demeanor a wide-ranging set of tryouts on 02 actual communal datasets and validate that NSPR suggestively beats the state-of-the-art standards.

In this investigation ${ }^{[72]}$, the authors suggested to prototypical user preferences and item properties, both expending a convolutional neural network $(\mathrm{CNN})$ with consideration, inspired by the enormous achievement of CNN for numerous natural language processing (NLP) errands. The authors authenticate the projected representations on prevalent evaluation datasets, subordinate significances thru matrix-factorization (MF), and hidden-factor-and-topical (HFT) representations. The experiments demonstration enhancement is done HFT, which demonstrates the efficiency of these illustrations learned from proposed networks on assessment text for rating estimation. The schematic diagram shows in Figure 3. 


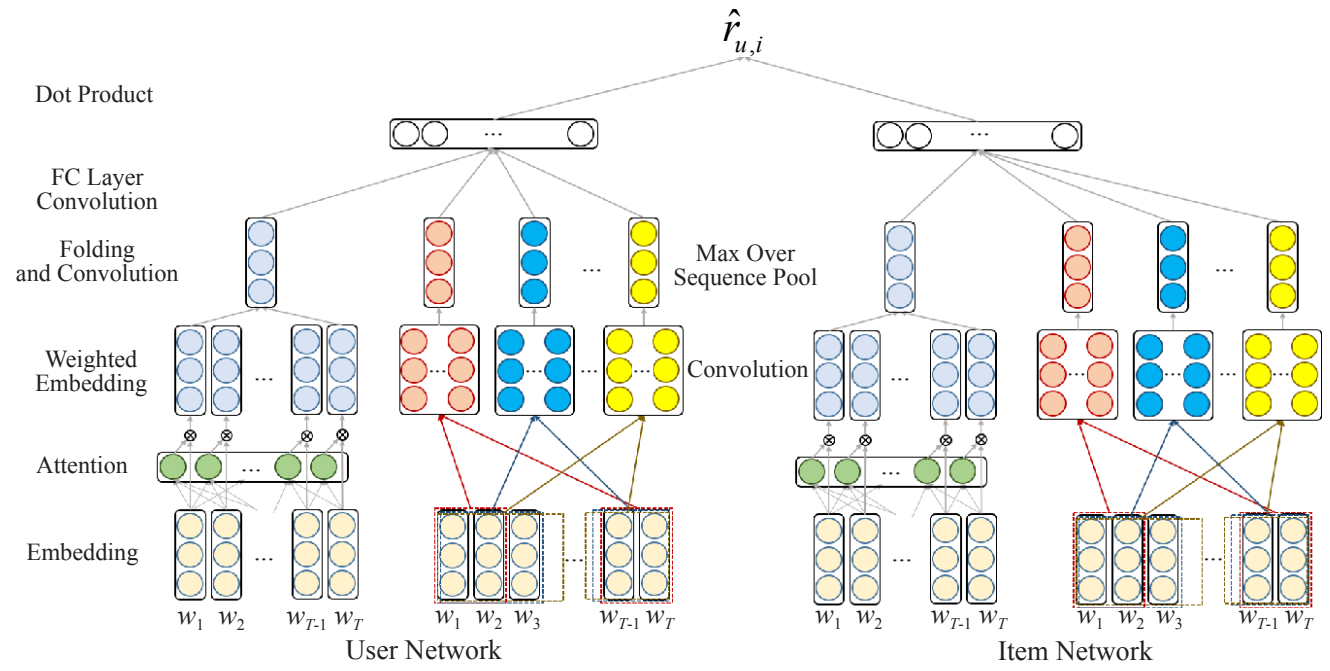

Figure 3. Attention-based CNNs to extract latent depictions of users or items, as divided in two components namely as left and right ${ }^{17}$

The above figure is divided into two sections, left most section contains the attention component whereas rightmost section contains the $\mathrm{CNN}$ component. Both sections are coupled together via $\mathrm{CNN}$ and fully connected layer for rating predictions ${ }^{[72]}$.

The author represents scalars thru $(x, y)$, vectors with $(\mathrm{x}, \mathrm{z})$ and matrices $(\mathrm{X}, \mathrm{W})$. The weight of the embedding layer is $W_{e} \in \mathfrak{R}^{d \times|v|}$ :

$$
X_{t}=W_{e} e_{t}
$$

and $v$ contains words, $|v|$ magnitude of the vocabulary shows 20,000 words.

In an Attention Component on the left section, the attention words $\left(\widehat{X}_{1}, \widehat{X}_{2}, \ldots, \widehat{X}_{T}\right)$ are crumpled over the sumoperation beside the progressive command, $y=\sum_{t} \widehat{X}_{t}$. And lastly, the attention demonstration gained via Convolution operation using matrix $W_{\text {att }}^{2} \in \mathfrak{R}^{d \times n_{\text {att }}}$ also a bias $b_{\text {att }}^{2} \in \mathfrak{R}^{n_{a t t}}$ :

$$
z_{\text {att }}(i)=g\left(y * W_{\text {att }}^{2}(:, i)+b_{\text {att }}^{2}(i)\right), i \in\left[1, n_{\text {att }}\right]
$$

$n_{\text {att }}$ is the number of filters and $g$ is a tanh function.

Convolutional component on the right section, the word sequence as of $D_{u}$ is input to the CNN component to learn a global semantic demonstration for $u$.

$$
\begin{aligned}
& X_{\text {conv }, i}=\left(X_{i}, X_{i+1}, \ldots, X_{i+\omega f-1}\right)^{T}, Z(i, j)=g\left(X_{\text {conv }, i} * W_{c o n v}(:,:, j)+b_{c o n v}(j)\right), \\
& i \in\left[1, T-w_{f}+1\right], j \in\left[1, n_{\text {conv }}\right] .
\end{aligned}
$$

$g$ is a nonlinear-activation function and $b_{\text {covn }}$ is a bias vector. In the pooling layer, a max pooling is smeared over the arrangement: $z_{\text {covn }}(j)=\operatorname{Max}(\mathrm{Z}(: ; j))$ And can attain as many: $z_{\text {covn }}$ as different filter length $w_{f}$.

Last layers, the outcome of the attention component and the $\mathrm{CNN}$ component are concatenated, and route over a further convolutional layer $W_{\text {out }}$ and $\mathrm{FC}$ and $W_{F C}$.

$$
\begin{aligned}
& z_{0}=z_{\text {att }} \oplus z_{\text {conv }}^{1} \oplus \ldots \oplus z^{\# n_{\text {conv }}}, \\
& z_{\text {out }}(i)=g\left(z_{0} * W_{\text {out }}(:, i)+b_{\text {out }}(i)\right), i \in\left[i, n_{\text {out }}\right], \gamma_{u}=W_{F C} \cdot z_{\text {out }} .
\end{aligned}
$$


$\oplus$ is a concatenation operator and $n_{\text {out }}$ is the number of filters applied to $z_{0}$.

In this paper ${ }^{[73]}$, the authors established a correspondence amongst the momentum stricture in the gradient descent learning procedures and the quantity of Newtonian particles that interchange through a viscous mediocre underneath an unadventurous strength meadow. The performance of gradient descent nearby a native least is comparable to a set of attached and curbed harmonic oscillators. Inside a sensible stricture variety, the momentum period can recover the speediness of convergence for greatest Eigen mechanisms in the structure by transporting them nearer to serious curbing. On behalf of the discrete-time circumstance, the momentum period delivers the extra assistance of approximate replication the stricture variety over which the structure congregates.

In this investigation ${ }^{[7]}$ the authors demonstrate, that a class named Restricted Boltzmann Machines (RBM's) which consists of two-layer, can be cast-off to prototypical tabular information, such as user's assessments of cinemas. The author's extant effectual learning and inference dealings with the class of demonstrations and corroborate that RBM's be able to efficaciously sensible to the Netflix-dataset, comprehending above billions of user/movie grades. The authors besides demonstrate that the separately RBM's and SVD representations are not able to predict better as compared they joint together.

The author used "softmax" designed for sculpting every column of the perceived "visible" binary assessment matrix $\mathrm{V}$ and a conditional Bernoulli distribution for modeling "hidden" user features $h$ for M movies, $\mathrm{N}$ users, and integer rating values from 1 to $\mathrm{K}$.

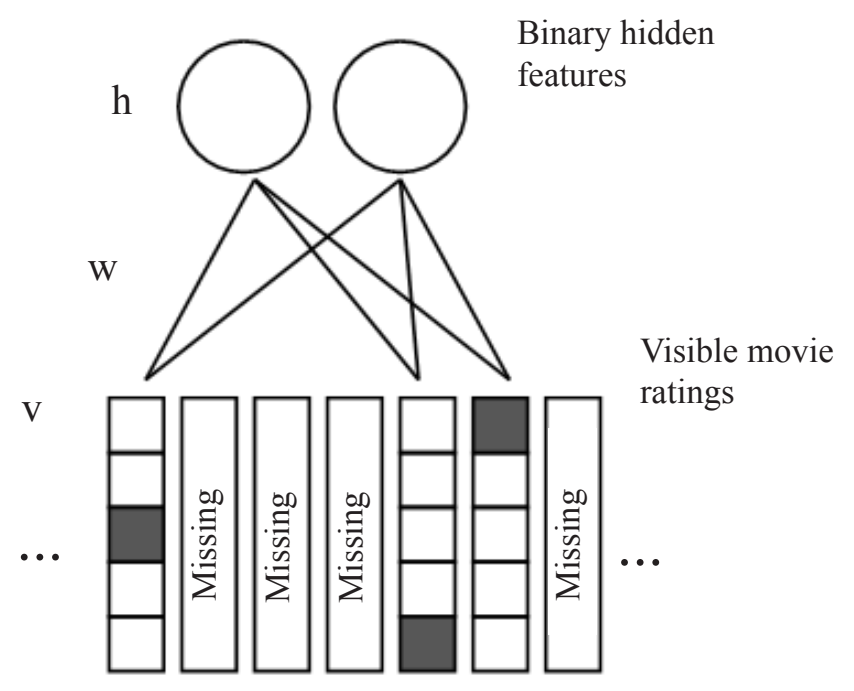

Figure 4. A restricted Boltzmann machine with binary hidden units and softmax visible units ${ }^{[7]}$

The marginal distribution over the visible ratings V:

$$
p(V)=\sum_{h} \frac{\exp (-E(V, h))}{\sum V^{\prime}, h^{\prime} \exp \left(-E\left(V^{\prime}, h^{\prime}\right)\right)}
$$

with an energy" term given by:

$$
E(V, h)=-\sum_{i=1}^{m} \sum_{j=1}^{F} \sum_{k=1}^{k} W_{i j}^{k} h_{j} v_{i}^{k}+\sum_{i=1}^{m} \log Z_{i}-\sum_{i=1}^{m} \sum_{k=1}^{K} v_{i}^{k} b_{i}^{k}-\sum_{j=1}^{f} h_{j} b_{j}
$$

The movies through omitted ratings do not make any influence on the energy function.

The parameter modernizes prerequisite to achieve gradient ascent in the log-likelihood can be gained from $p(V)$.

$$
\Delta W_{i j}^{k}=\epsilon \frac{\partial \log p(V)}{\partial W_{i j}^{k}}=\epsilon\left(\left\langle v_{i}^{k} h_{j}\right\rangle \text { data }-\left\langle v_{i}^{k} h_{j}\right\rangle \text { model }\right)
$$


where $\in$ is the learning rate.

Particular the observed ratings $\mathrm{V}$, can predict a rating for a novel query movie $q$ in time linear in the number of hidden units:

$$
\begin{aligned}
& p\left(v_{q}^{k}=1 \mid V\right) \propto \sum_{h_{1}, \ldots, h_{p}} \exp \left(-E\left(v_{q}^{k}, V, h\right)\right) \\
& \propto \tau_{q}^{k} \prod_{j=1}^{F} \sum_{h_{j} \in\{0,1\}} \exp \left(\sum_{i l} v_{i}^{l} h_{j} W_{i j}^{l}+v_{q}^{k} h_{j} W_{q j}^{k}+h_{j} b_{j}\right) \\
& =\tau_{q}^{k} \prod_{j=1}^{F}\left(1+\exp \left(\sum_{i l} v_{i}^{l} h_{j} W_{i j}^{l}+v_{q}^{k} h_{j} W_{q j}^{k}+b_{j}\right)\right)
\end{aligned}
$$

By expending the over-parametrization of the softmax, the RBM can learn to use omitted ratings to stimulus its hidden features, even though it does not try to reconstruct these omitted ratings and it does not accomplish any reckonings that scale with the number of omitted ratings. The conditional RBM model takes this further data into account ${ }^{[74]}$.

In this study ${ }^{[75]}$, the authors suggested the usage of a latent factor prototypical for RS, and forecast the latent factors commencing music-audio while they cannot be gotten commencing custom information. The authors equate an oldfashioned tactic by means of a bag-of-words with DCNN used for specific datasets for predictions.

The authors demonstrate that exhausting forecast latent factors yield workable recommendations, notwithstanding the circumstance that around is a huge semantic breach amongst the features of a song that shake user predilection and the equivalent audio indication. The authors moreover illustration that modern improvements in DL interpret very healthy to the music recommendation background, by DCNN suggestively beating the customary tactic.

The author used the weighted matrix factorization (WMF) algorithm, to learn latent factor depictions of each users and items in the Taste-Profile Subset. This is a modified matrix factorization algorithm designed at inherent feedback datasets.

Let $r_{u i}$ be the play count for user $u$ and song $i$. For each user-item pair, we define a preference variable $p_{u i}$ and a confidence variable $c_{u i} I(X)$ is the indicator function, $\propto$ and $\in$ are hyper parameters):

$$
\begin{aligned}
& p_{u i}=I\left(r_{u i}>0\right), \\
& c_{u i}=1+\propto \log \left(1+\epsilon^{-1} r_{u i}\right)
\end{aligned}
$$

The predilection variable designates whether user $u$ has ever listened to song $i$. If it is 1 , the author presumed the user likes the song. The confidence variable dealings how certain about this specific preference. It is a function of the play count because songs with higher play counts are more likely to be favoured. If the song has never been played, the confidence variable will have a short value, because this is the slightest instructive case.

The WMF objective function is given by:

$$
\min _{x^{*}, y^{*}} \sum_{u, i} c_{u i}\left(p_{u i}-x_{u}^{T} y_{i}\right)^{2}+\lambda\left(\sum_{u}\left\|x_{u}\right\|^{2}+\sum_{i}\left\|y_{i}\right\|^{2}\right)
$$

Latent factor vectors achieved by smearing WMF to the obtainable usage data are cast-off as ground truth to train the forecast models.

In this study ${ }^{[76]}$, the authors proposed co-occurrence information expending a broad energy-based probabilistic prototypical, and authors examine 03 dissimilar kinds of energy-based prototypical, viz., the $L_{1}, L_{2}$ and $L_{k}$ representations, which are intelligent to apprehension diverse stages of addiction in the co-occurrence information. The authors moreover deliberate how numerous distinctive prevailing representations are connected to these 03 kinds of energy representations, with the Fully Visible Boltzmann Machine (FVBM) $\left(L_{2}\right)$, Matrix Factorization $\left(L_{2}\right)$, Log-BiLinear (LBL) models $\left(L_{2}\right)$, and the Restricted Boltzmann Machine (RBM) model $\left(L_{k}\right)$. Then, for this purpose, authors proposed a Deep Embedding Model 
(DEM) (an Lk model) as of the energy model in a righteous way. Finally, in the practical investigation, indicated that the DEM could attain analogous or improved consequences than state-of-the-art approaches on datasets transversely numerous application provinces.

The author present 03 Bayesian dependence conventions, namely, $L_{1}, L_{2}$ and $L_{k}$ on the model, where the energy utility $E_{\theta}(v)$ would assume different forms under different conventions. Inside this background, the author shows that numerous prevalent statistical facsimiles fall into distinct groups of the background, and also enlighten how different categories of representations are able to trade prototypical capability with model density.

Study Bayesian $L_{1}$ Dependence Hypothesis, where the items in co-occurrence data are expected to be self-governing of separately so that the probability mass function of $v$ can be factorized into the following product form:

$$
p_{\theta}(v)=\prod_{i \in I_{v}} p(i) \prod_{i \notin I_{v}}(1-p(i))
$$

Where $I_{v}$ denotes the set of the items occurred in $v$, and $p(i)$ is the occurrence probability of the i-th item.

For Bayesian $L_{2}$ Dependence Hypothesis Likewise, for the Bayesian $L_{2}$ dependence, the energy function $E_{\theta}(v)$ in assumes the following form:

$$
E_{\theta}^{L_{2}}(v)=v^{T} W v+b^{T} v
$$

Where $\mathrm{W}$ is a $N \times N$ symmetric matrix with zero diagonal entries.

The Bayesian $L_{k}$ dependence hypothesis anticipated to model any high-order correlations between items in cooccurrence tasters. Thus, the author extends the classical $L_{2}$ FVBM model with $L_{k}$ FVBM. The new energy function for $L_{k}$ FVBM could be given as follows:

$$
E_{\theta}^{L_{k}}(v)=\sum_{i \in I_{v}} b_{i}+\sum_{i, j \in I_{v}(i \neq j)} W_{i j}+\ldots+\sum_{i, j, \ldots, k \in I_{v}(i \neq j . . \neq k)} W_{i j . k}
$$

The dynamic energy function for the deep embedding model is given by,

$$
F_{\theta}^{D E M}(t, v)=b_{t}+\sum_{i \in I_{v}} R_{i t}^{0}+R_{t}^{1} h_{1}+R_{t}^{2} h_{2}+\ldots+R_{t}^{k} h_{k}
$$

The efficiency of the Deep Embedding Model (DEM) empirically on numerous real world datasets. The datasets are classified into 03 fields: Social networks, Product Co-Purchasing and Online-Rating Data.

In this paper author talked about the sparsity ${ }^{[77]}$ problem, collaboratively topic regression (CTR) are jointly 02 devices that learn with 02 different data. In this study, the author's usages a hierarchical-Bayesian-model called collaborativeDL (CDL), which equally learns the competent data. Widespread on practically shows that the 03 actual datasets are commencing diverse field's spectacle that CDL can suggestively improvement the state-of-the-art approaches.

Matrix factorization (MF) ${ }^{[78]}$ models and their postponements are customary in recent RS. MF representations crumble the pragmatic user-item collaboration matrix into user and item latent factors. In this investigations, the authors intend a co-factorization prototypical, Cofactor, which cooperatively crumbles the user-item collaboration matrix and the item-item co-occurrence matrix with communal item latent factors. The authors suggested that this kind of mutual factorization harvests recital enhancements in recommendation metrics in a variety of surroundings: recommending documents to investigators on ArXiv, movies on MovieLens, and music on Taste Profile. The authors recognize the set-ups wherever Cofactor beats standard MF, which elasticities advance visions into the assistances of 'reusing' the information. The authors demonstrate that regularizing by means of item co-occurrence sums allows Cofactor to recommend rare substances by apprehending their co-occurrence decorations, while this feature is inattentive in typical MF. The author's opinion to the probable of substitute approaches such as regularizing with user-user co-occurrence or in the situation of other widely-used CF models.

In this study ${ }^{[79]}$, the authors presented a NN construction, aka CFN, to accomplish CF with side data. Conflicting to other efforts by NN, this dual Network assimilates side data and studies a non-linear depiction of users or items into an exclusive NN. This tactic is able to strokes state of the art consequences in CF on together MovieLens and Douban 
datasets. It executes exceptional consequences in together cold-start and warm-start situation. CFN has also appreciated properties for manufacturing, it is accessible, healthy, and it efficaciously contracts with the great dataset. The authors conclude that refillable source code is delivered in Torch and hyper parameters are delivered to imitate the outcomes. The author's illustration practically on the MovieLens and Douban dataset that CFN beats state of the art and reimbursements commencing side evidence.

In this investigation ${ }^{[80]}$ authors firstly suggested old-fashioned methods elaborate in RS and DL, and then a broad survey and Criticism of numerous state-of-the-art deep RS. Totally, owed to the restriction of the outmoded recommendation tactics, the probable of comfortable data has not been entirely demoralized. By the assistance of the gain of DL in demonstrating diverse kinds of information, deep recommender systems can healthier comprehend what customers prerequisite and further expand recommendation quality?

The author designed Trigger-and-Trigger (TT) framework ${ }^{[81]}$ for concentrated bias problems, the TT couple via significant correlation and is used for experimental unidentified recommendation. Experimental validation showed the success of the proposed framework as a real time retail store information.

The author described advertisement-clicking problem ${ }^{[82]}$ via 04 multiple criteria mathematical programming facsimiles.Click-through-rate (CTR) forecast used multi-criteria-linear-regression (MCLR) and kernel-based-multiplecriteria-regression (KMCR) processes, whereas for predicting the ads multi-criteria-linear-programming (MCLP) and kernel-based-multiple-criteria-programming (KMCP) processes are used. The extensive experiments shows that the MCLP and KMCP prototypes have superior performance immovability and can be cast-off to efficiently grip over interactive steering application for online advertisement glitches.

\section{Conclusion}

DL has come to be more widespread in all subfields of Computer Science and applications, like NLP, image and video processing, computer / machine vision, Digital Image Processing etc. These methods have consistently managed to attract scientists with new approaches, which is capable of solving any kind of difficulties.

DL is not even extremely accomplished of curing multifaceted difficulties in numerous arenas. Nonetheless, they likewise formed a communal lexis and shared crushed for these investigation pitches. Deep learning helps in all fields, where previously, it was difficult to crack the complications.

The objective of this research is that all the research that has been done so far in the Deep Learning-based recommended system can be made available to the researchers so that more work can be done in the shortest possible time in the coming time. The time to come will be deep learning. It has also been proved by research. Therefore the greatest difficulty in this job is to handle the large volume of data and cold-start problem. Therefore, different issues and challenges have also been told in this work. Deep learning is a huge network in itself. We just have to find a better approach to specific problems for this, if we take two or more methods together instead of a single method, it can give better results, which is called fusion or hybrid method, as the researchers have proved from their research work.

\section{References}

[1] Aggarwal CC. Content-based recommender systems. Recommender systems. Berlin: Springer; 2016. p.139-166.

[2] Ekstrand, MD, Riedl J, et al. Collaborative filtering recommender systems. Foundations and Trends in Human-Computer Interaction. 2014; 175-243.

[3] Lian J, Zhou X, Zhang F, et al. xDeepFM: Combining explicit and implicit feature interactions for recommender systems. Proceedings of the 24th ACM SIGKDD International Conference on Knowledge Discovery \& Data Mining. 2018.

[4] Jannach D, Zanker M, Felfernig A, et al. Recommender systems-an introduction. 2010.

[5] Ricci F, Rokach L, Shapira B. Recommender systems: Introduction and challenges. In Recommender systems handbook. 2015; 1-34.

[6] Wang N, Yeung DY. Learning a deep compact image representation for visual tracking. In NIPS. 2013; 809-817.

[7] Kalchbrenner N, Grefenstette E, Blunsom P. A convolutional neural network for modelling sentences. ACL. 2014; 655-665.

[8] Salakhutdinov R, Mnih A. Probabilistic Matrix Factorization. NIPS. 2007.

[9] Nguyen TV, Karatzoglou A, Baltrunas L. Gaussian process factorization machines for context-aware recommendations. In Proceedings of the 37th international ACM SIGIR conference on Research \& development in information 
retrieval. 2014. p.63-72.

[10] Hidasi B. Context-aware factorization methods for implicit feedback based recommendation problems. PhD thesis. Hungary: Budapest University of Technology and Economics; 2016.

[11] Hidasi B. Factorization models for context-aware recommendations. Infocommun J. 2014; 6(4): 27-34.

[12] Hidasi B, Tikk D. General factorization framework for context-aware recommendations. Data Mining and Knowledge Discovery. 2016; 30(2): 342-371.

[13] Hidasi B, Tikk D. Fast ALS-based tensor factorization for context-aware recommendation from implicit feedback. Machine Learning and Knowledge Discovery in Databases. 2012; 67-82.

[14] Karatzoglou A, Amatriain X, Baltrunas L, et al. Multiverse recommendation: n-dimensional tensor factorization for context-aware collaborative filtering. In Proceedings of the fourth ACM conference on Recommender systems. 2010. p.79-86.

[15] Baltrunas L, Ludwig B, Ricci F. Matrix factorization techniques for context aware recommendation. In Proceedings of the fifth ACM conference on Recommender systems. 2011. p.301-304.

[16] Rendle S, Gantner Z, Freudenthaler C, et al. Fast context-aware recommendations with factorization machines. In Proceedings of the 34th international ACM SIGIR conference on Research and development in Information Retrieval. 2011. p.635-644.

[17] Rendle S, Schmidt-Thieme L. Pairwise interaction tensor factorization for personalized tag recommendation. In Proceedings of the third ACM international conference on Web search and data mining. 2010. p.81-90.

[18] Rendle S, Freudenthaler C, Gantner Z, et al. BPR: Bayesian personalized ranking from implicit feedback. In Proceedings of the twenty-fifth conference on uncertainty in artificial intelligence. AUAI Press; 2009. p.452-461.

[19] Burke R. Hybrid web recommender systems. In Brusilovsky, P., Kobsa, A. and Nejdl, W. (eds). The Adaptive Web. Berlin, Heidelberg: Springer; 2007. p.377-408.

[20] Ricci F, Rokach L, Shapira B. Kantor Recommender Systems Handbook. P.B. (Eds.). first ed. XXX. $2011 ; 842: 20$.

[21] Su X, Khoshgoftaar TM. A survey of collaborative filtering techniques. Advances in Artificial Intelligence. 2009; 19: 421425.

[22] Yang X, Guo Y, Liu Y, et al. A survey of collaborative filtering based social recommender systems. Elsevier, Computer Communications. 2014; 1-10. Available from: http://dx.doi.org/10.1016/j.comcom.2013.06.009.

[23] Karypis G. Evaluation of item-based top-N recommendation algorithms. Proc. 10th Int. Conf. Information and Knowledge Management. 502627 ACM. USA: New York; 2011. p.247-254.

[24] Xue G R, Lin C, Yang Q, et al. Scalable collaborative filtering using cluster based smoothing. Proc. 28th Annual Int. ACM SIGIR Conference on Research and Development in Information Retrieval. 2005. p.114-121.

[25] Aggarwal CC. Model-based collaborative filtering. In Recommender Systems: The Textbook. Springer International Publishing, Cham; 2016. p.71-138.

[26] Wang Y, Chan SCF, Ngai G. Applicability of demographic recommender system to tourist attractions: A case study on trip advisor. Proc. 2012 IEEE/WIC/ACM Int. Joint Conf. Web Intelligence and Intelligent Agent Technology. EEE Computer Society, Washington, DC, USA; 2012. p.97-101.

[27] Burke R. Hybrid recommender systems: Survey and experiments. User Model and User-adapt. Interact. 2004; 12: 331-370.

[28] Adomavicius G, Tuzhilin A. Context-aware recommender systems. In Ricci F, Rokach, L, Shapira B and Kantor P B (eds). Recommender Systems Handbook. Springer; 2011. p.217-253.

[29] Morris MR, Teevan J, Panovich K. What do people ask their social networks, and why?: A survey study of status message q \& a behavior. Proc. SIGCHI Conf. Human Factors in Computing Systems. ACM New York USA; 2010. p.1739-1748.

[30] Katarya R. A systematic review of group recommender systems techniques. Int. Conf. Intell. Sustain. Syst. 2017; 425428.

[31] Khan MM, Ibrahim R, Ghani I. Computing. Cross domain recommender systems: A systematic literature review. $A C M$ Comput. Surv. 2017; 50: 1-34.

[32] Kumar N. A survey on data mining methods available for recommendation system. 2nd Int. Conf. Comput. Syst. Inf. Technol. Sustain. Solution. 2017. p.1-6.

[33] Garzó A. Cross-lingual web spam classification. Int. World Wide Web Conf. Comm. 2013. p.1149-1156.

[34] Ferdous SN, Ali MM. A semantic content based recommendation system for cross-lingual news. IEEE International Conference on Imaging, Vision \& Pattern Recognition (icIVPR). 2017. p.1-6.

[35] Almazro D, Shahatah G, Albdulkarim L, et al. A Survey Paper on Recommender Systems. 2010. Available from: ArXiv, abs/1006.5278.

[36] Pronk V, Verhaegh W, Proidl A, et al. Incorporating user control into recommender systems based on naive bayesian classification. In RecSys 07. Proceedings of the 2007 ACM conference on Recommender systems. 2007. p.73-80. 
[37] Li L, Wang D, Li T, et al. Scene: A scalable two-stage personalized news recommendation system. In SIGIR '11 Proceedings of the 34th international ACM SIGIR conference on Research and development in Information Retrieval. 2011. p.125-134.

[38] Fortuna B, Fortuna C, Mladenic D. Realtime news recommender system. In ECML PKDD'10 Proceedings of the European conference on Machine learning and knowledge discovery in databases: Part III. 2010. p.583-586.

[39] Liu J, Dolan P, Pedersen ER. Personalized news recommendation based on click behavior. In IUI'10 Proceedings of the 15th international conference on intelligent user interfaces. 2010. p.31-40.

[40] Saranya KG, Sadhasivam G. A personalized online news recommendation system. International Journal of Computer Applications. 2012; 57(18): 6-14.

[41] Das A, Datar M, Garg A, et al. Google news personalization: Scalable online collaborative filtering. In WWW'07 Proceedings of the 16th international conference on World Wide Web. 2007. p.271-280.

[42] Su X., Khoshgoftaar TM. A survey of collaborative filtering techniques. Advances in artificial intelligence. 2009.

[43] Borges HL, Lorena AC. A survey on recommender systems for news data. In Smart Information and Knowledge Management, Springer. 2010; 129-151.

[44] Iaquinta L, Gemmis MD, Lops P, et al. Introducing serendipity in a content-based recommender system. In Hybrid Intelligent Systems, HIS'08. Eighth International Conference. IEEE. 2008. p.168-173.

[45] G Srinivasa PSS, Archana M. Survey paper on recommendation system using data mining techniques. Int. J. Eng. Comput. Sci. 2016; 6(4): 2454-4698.

[46] Patel YG, Patel VP. A survey on various techniques of personalized news recommendation system. Int. J. Eng. Dev. Res. 2015; 3(4): 696-700.

[47] Oord AV, Dieleman S, Schrauwen B. Deep content-based music recommendation. NIPS. 2013.

[48] Li S, Kawale J, Fu Y. Deep collaborative filtering via marginalized denoising auto-encoder. CIKM. 2015.

[49] Liang D, Zhan M, Ellis DP. Content-aware collaborative music recommendation using pre-trained neural networks. ISMIR. 2015.

[50] Strub F, Mary J. Collaborative filtering with stacked denoising auto encoders and sparse inputs. NIPS. 2015.

[51] Almahairi A, Kastner K, Cho K, et al. Learning distributed representations from reviews for collaborative filteringg. 2015. Available from: ArXiv abs/1806.06875.

[52] Elkahky AM, Song Y, He X. A multi-view deep learning approach for cross domain user modeling in recommendation systems. $W W W .2015$.

[53] Ko Y, Maystre L, Grossglauser M. Collaborative recurrent neural networks for dynamic recommender systems. ACML. 2016.

[54] Bansal T, Belanger D, McCallum A. Ask the GRU: Multi-task learning for deep text recommendations. RecSys. 2016.

[55] Strub F, Mary J, Gaudel R. Hybrid collaborative filtering with neural networks. 2016. Available from: ArXiv abs/1603.00806.

[56] Strub F, Mary J, Gaudel R. Hybrid recommender system based on autoencoders. 2016. Available from: ArXiv abs/1606.07659.

[57] Vasile F, Smirnova E, Conneau A. Meta-prod2vec: Product embeddings using side-information for recommendation. 2016. Available from: ArXiv abs/1607.07326.

[58] Cheng H, Koc L, Harmsen J, et al. Wide \& deep learning for recommender systems. DLRS. 2016.

[59] Chiliguano P, Fazekas G. Hybrid music recommender using content-based and social information. IEEE International Conference on Acoustics, Speech and Signal Processing (ICASSP). 2016. p.2618-2622.

[60] Hidasi B, Karatzoglou A, Baltrunas L, et al. Session-based recommendations with recurrent neural networks. 2015. Available from: CoRR abs/1511.06939.

[61] Zheng Y, Tang B, Ding W, et al. A neural autoregressive approach to collaborative filtering. 2016. Available from: ArXiv abs/1605.09477.

[62] Covington P, Adams JL, Sargin E. Deep neural networks for YouTube recommendations. RecSys. 2016.

[63] Hidasi B, Quadrana M, Karatzoglou A, et al. Parallel recurrent neural network architectures for feature-rich session-based recommendations. RecSys. 2016.

[64] Tan Y K, Xu X, Liu Y. Improved recurrent neural networks for session-based recommendations. 2016. Available from: ArXiv abs/1606.08117.

[65] Balakrishnan, A. Deepplaylist: Using recurrent neural networks to predict song similarity. Conference proceeding Stanford. 2016.

[66] Wu Y, DuBois C, Zheng AX, et al. Collaborative denoising auto-encoders for top-N recommender systems. WSDM. 2016.

[67] Zheng L, Noroozi V, Yu PS. Joint deep modeling of users and items using reviews for recommendation. WSDM. 2017.

[68] Trofimov M, Sidana S, Horodnitskii O, et al. Representation learning and pairwise ranking for implicit and explicit 
feedback in recommendation systems. 2017. Available from: ArXiv abs/1705.00105.

[69] Catherine R, Cohen WW. TransNets: Learning to transform for recommendation. RecSys. 2017.

[70] Pan Y, He F, Yu H. Trust-aware collaborative denoising auto-encoder for top-N recommendation. Information Science Journal. Arxiv. 2017.

[71] Ebesu T, Fang Y. Neural semantic personalized ranking for item cold-start recommendation. Information Retrieval Journal. 2017; 109-131.

[72] Seo S, Huang J, Yang H, et al. Representation learning of users and items for review rating prediction using attention-based convolutional neural network. 2017.

[73] Qian N. On the momentum term in gradient descent learning algorithms. Neural networks: the official journal of the International Neural Network Society. 1999; 145-151.

[74] Salakhutdinov R, Mnih A, Hinton G E. Restricted boltzmann machines for collaborative filtering. ICML. 2007.

[75] Oord AV, Dieleman S, Schrauwen B. Deep content-based music recommendation. NIPS. 2013.

[76] Shen Y, Jin R, Chen J, et al. A deep embedding model for co-occurrence Learning. IEEE International Conference on Data Mining Workshop (ICDMW). 2015. p.631-638.

[77] Wang H, Wang N, Yeung D. Collaborative deep learning for recommender systems. KDD. 2014.

[78] Liang D, Altosaar J, Charlin L, et al. Factorization meets the item embedding: regularizing matrix factorization with item co-occurrence. RecSys. 2016.

[79] Strub F, Mary J, Gaudel R. Hybrid collaborative filtering with autoencoders. ArXiv. 2016.

[80] Zheng L. A survey and critique of deep learning on recommender systems. 2016.

[81] Deng W, Shi Y, Chen Z, et al. Recommender system for marketing optimization. The World Wide Web Journal. 2020; 23: 1497-1517.

[82] Lee J, Shi Y, Wang F, et al. Advertisement clicking prediction by using multiple criteria mathematical programming. The World Wide Web Journal. 2016; 19(4): 707-724. 